\title{
INTRODUZIONE ALLA TEORIA DEL MASSIMO COMUN DIVISORE E DEL MINIMO COMUNE MULTIPLO \\ DI PIÙ FUNZIONI ALGEBRICHE INTERE DI UNA SOLA VARIABILE.
}

\author{
Nota di Giovanni Z. Giambelli (Torino).
}

Adunanza del 28 luglio 1907 .

La ricerca delle condizioni, affinchè $r$ equazioni ad una incognita abbiano più radici in comune, è stata trattata in modo completo solo quando $r=2$, e non si conosce alcun risultato notevole pel caso di $r>2^{\mathbf{x}}$ ). Nella presente Nota si tratterà tale questione generale, ossia il problema:

Determinare le condizioni necessarie e sufficienti, affinchè più funzioni algebriche intere di una sola variabile comunque prese ad $u$ ad $u$ ammettano M.C.D. e M.C.M. di dati $\operatorname{gradi}^{2}$ ).

Di questo problema, che si puó pensare come una introduzione alla teoria del M.C.D. e del M.C.M. di più funzioni algebriche intere di una sola variabile, si sono trovate nel $\$ 4$ due soluzioni, conseguenze immediate di alcune questioni ausiliarie trattate nei $\int \ \mathrm{I}, 2,3$. Tra i risultati di questi $\$\lceil$ vi è un nuovo modo di enunciare la condizione necessaria e sufficiente, affinchè due equazioni abbiano più radici in comune, considerando l'annullamento di una matrice simile a quella ricavata dal determinante di Sylvester (o di Eulero), i cui elementi peró sono le funzioni aleph di Wronski delle radici delle due equazioni. Inoltre sono state trovate le formole per la costruzione del M.C.D. e del M.C.M. di due, o più, funzioni algebriche intere di una sola variabile ${ }^{3}$ ).

$$
\int I \text {. }
$$

\section{Definizioni. - Proposizioni e formole ausiliarie.}

Si designi con:

$S_{i}(i=\mathrm{I}, 2, \ldots, s+\mathrm{I})$ la funzione simmetrica fondamentale $\sum x_{0} x_{\mathrm{i}} \ldots x_{i-1}$ di grado $i$ nelle $x_{0}, x_{1}, \ldots, x_{s}$;

r) Cfr. l'Articolo: Rationale Funktionen einer Veränderlichen; ihre Nullstellen, di E. NETro, nella "Encyklopädie der Mathematischen Wissenschaften", Band I, Teil I (I898), pp. 227-254.

2) M.C.D. e M.C.M. significano rispettivamente massimo comun divisore e minimo comune multiplo.

3) Dovendo spesso ricordare alcuni risultati della mia Nota: Alcune proprietà delle funzioni simmetriche caratteristiche [Atti della R. Accademia delle Scienze di Torino, vol. XXXVIII (1903), pp. 823-844], si designerà col simbolo $\mathfrak{R}$ tale Nota. 
$V_{i}(i=1,2,3, \ldots)$ la funzione aleph di ordine $i$ delle $x_{0}, x_{1}, \ldots, x_{1}$, ossia la funzione simmetrica delle $x_{0}, x_{1}, \ldots, x_{s}$, ottenuta dallo sviluppo $\left(x_{0}+x_{1}+\cdots+x_{s}\right)^{i}$, quando in luogo di ciascuno dei coefficienti polinomiali si ponga l'unità.

Si convenga di porre:

$$
\begin{aligned}
& S_{0}=V_{0}=\mathrm{I}, \\
& S_{i}=V_{i}=0 \text {, se } i \text { è negativo, } \\
& S_{i}=0, \text { se } i \text { è maggiore di } s+\mathrm{I} .
\end{aligned}
$$

Particolarizzando rispettivamente la (4) del $\ 2$ e la (6) del $\ 3$ di $\mathfrak{R}$ si ottengono facilmente le formole:

$$
\begin{aligned}
V_{i}=\sum_{u=0}^{u=i=1}(-\mathrm{I})^{i-u-1} V_{u} \cdot S_{i-u} & (i=1,2,3, \ldots), \\
S_{i}=\sum_{u=0}^{u=i-1}(-I)^{i-u-1} S_{u} \cdot V_{i-u} & (i=1,2,3, \ldots) .
\end{aligned}
$$

Si consideri poi il gruppo delle $t+$ I lettere $x_{0}^{\prime}, x_{1}^{\prime}, \ldots, x_{1}^{\prime}$ e si designi con:

$T_{i}(i=1,2, \ldots, t+1)$ la funzione simmetrica fondamentale di grado $i$ nelle $x_{0}^{\prime}, x_{1}^{\prime}, \ldots, x_{i}^{\prime}$;

$W_{i}(i=1,2,3, \ldots)$ la funzione aleph di ordine $i$ nelle $x_{0}^{\prime}, x_{1}^{\prime}, \ldots, x_{i}^{\prime}$;

colla convenzione di porre:

$$
\begin{aligned}
& T_{\mathrm{o}}=W_{\mathrm{o}}=\mathrm{I}, \\
& T_{i}=W_{\mathrm{i}}=\mathrm{o}, \text { se } i \text { è negativo, } \\
& T_{i}=\mathrm{o}, \text { se } i \text { è maggiore di } t+\mathrm{I} .
\end{aligned}
$$

Supposto $0 \leq p \leq \min (s, t)$, si designino inoltre rispetrivamente con $X_{i}, Y_{i}$ le $S_{i}, V_{i}$, quando si faccia $x_{p+1}=x_{p+2}=\cdots=x_{s}=0$, e si designino rispettivamente con $(S-X)_{i},(V-Y)_{i}$ le $S_{i}, V_{i}$, quando si faccia $x_{\circ}=x_{i}=\cdots=x_{p}=0$.

\section{Evidentemente}

$$
\begin{aligned}
X_{0} & =Y_{0}=(S-X)_{0}=(V-Y)_{0}=\mathrm{r}, \\
X_{i} & =Y_{i}=(S-X)_{i}=(V-Y)_{i}=0, \text { se } i \text { è negativo, } \\
X_{i} & =0, \text { se } i \text { è maggiore di } p+\mathrm{I}, \\
(S-X)_{i} & =0, \text { se } i \text { è maggiore di } s-p .
\end{aligned}
$$

$\mathrm{Da}$ queste definizioni si ottengono subito le formole:

$$
\begin{aligned}
S_{i}=\sum_{u=0}^{u=i} X_{u} \cdot(S-X)_{i-u} & (i=0,1,2, \ldots), \\
V_{i}=\sum_{k=0}^{u=i} Y_{u} \cdot(V-Y)_{i-u} & (i=0,1,2, \ldots) .
\end{aligned}
$$

Eliminando le $X_{u}(i=0, \mathrm{I}, \ldots, i-\mathrm{r})$ tra le prime $i+\mathrm{r}$ formole (3) ed applicando la formola di P. GORDAn sulle funzioni simmetriche caratteristiche (ossia la formola duale di quella del Trudr, cfr. il teorema enunciato alla fine del $\int 5$ di $\Re$ ) rispetto alle $(V-Y)_{u}(u=\mathrm{I}, 2, \ldots, i)$, e similmente eliminando le $Y_{u}(u=0, \mathrm{I}, \ldots, i-\mathrm{I})$ 
tra le prime $i+\mathrm{I}$ formole (4) ed applicando la formola del Trudi (cfr. pure il $S \mathrm{~s}$ di $\mathfrak{R}$ ) rispetto alle $(S-X)_{u}[u=1,2, \ldots, \min (i, s-p)]$, si ricavano le formole inverse delle (3) e delle (4), ossia :

$$
\begin{array}{ll}
X_{i}=\sum_{u=0}^{u=i}(-1)^{i-u} S_{u} \cdot(V-Y)_{i-u} \quad(i=0,1,2, \ldots), \\
Y_{i}=\sum_{u=0}^{u=i}(-1)^{i-u} V_{u} \cdot(S-X)_{i-u} & (i=0,1,2, \ldots) .
\end{array}
$$

Ricordando che $0 \leqslant p \leqslant \min (s, t)$, si designi con :

$M(S, T ; s, t, p)$ la matrice di $s+t+2-2 p$ linee e di $s+t+2-p$ colonne nella quale gli elementi della colonna $(i+1)^{\text {sima }}(i=0, I, \ldots, s+t+1-p)$ sono:

$$
S_{i}, \quad S_{i-1}, \ldots, S_{i-i+p}, \quad T_{i}, \quad T_{i-r}, \ldots, T_{i-s+p} ;
$$

$M(V, W ; s, t, p)$ la matrice di $s+t+2-2 p$ linee e di $s+t+2-p$ colonne, nella quale gli elementi della colonna $(i+1)^{\operatorname{sima}}(i=0, I, \ldots, s+t+1-p)$ sono:

$$
V_{i}, \quad V_{i-1}, \ldots, V_{i-s+p}, \quad W_{i}, \quad W_{i-1}, \ldots ; W_{i-t-p} .
$$

Colla locuzione "matrice nulla» s'intende che siano nulli tutti i determinanti di ordine massimo contenuti nella matrice.

E importante dimostrare:

Trorema I. - Supposto $0 \leqslant p<\min (s, t)$, se è nulla la matrice $M(V, W ; s, t, p)$ e non è nulla la matrice $M(V, W ; s, t, p+1)$, allora è di conseguenza nulla la matrice $M(S, T ; s, t, p)$ e non è nulla la matrice $M(S, T ; s, t, p+1)$; supposto $p=\min (s, t)$, se è nulla la matrice $M(V, W ; s, t, p)$, allora $\dot{e}$ di conseguenza nulla la matrice $M(S, T ; s, t, p)$. Valgono le proprietà inverse.

$\dot{E}$ sufficiente dimostrare solo il teorema diretto, essendo facilissimo modificare la seguente dimostrazione per le proprieta inverse.

Se per convenzione si attribuisce il valore zero

$$
\begin{aligned}
& \text { alle } \sigma_{u} \text { per cui } u \gg s-p+\mathrm{I}, \\
& \text { alle } \tau_{u} \text { per cui } u \supseteq t-p+\mathrm{I},
\end{aligned}
$$

per l'ipotesi che è nulla la $M(V, W ; s, t, p)$ segue che il sistema delle $s+t+2-p$ equazioni

$$
\sum_{u=0}^{u-i} V_{u} \cdot \sigma_{i-u}=\sum_{u=0}^{u-i} W_{u} \cdot \tau_{i-u} \quad(i=0, x, \ldots, s+t+1-p)
$$

è soddisfarto da valori non tutti nulli delle incognite omogenee $\sigma_{0}, \sigma_{1}, \ldots, \sigma_{s-p}$, $\tau_{0}, \tau_{1}, \ldots, \tau_{t-p}$. Inoltre saranno diverse da zero almeno una delle $\sigma_{0}, \sigma_{1}, \ldots, \sigma_{1-p}$ ed almeno una delle $\tau_{0}, \tau_{1}, \ldots, \tau_{t-p}$, altrimenti sarebbero nulle tutte le $\sigma_{0}, \sigma_{1}, \ldots, \sigma_{1-p}$ e tutte le $\tau_{0}, \tau_{1}, \ldots, \tau_{1-p}$. Quindi quando è $p=\min (s, t)$, si ottiene subito $\sigma_{0}=\tau_{0} \neq 0$; ma anche se $\dot{e} p<\min (s, t)$, deve essere $\sigma_{0}=\tau_{0} \neq 0$, perchè se fosse $\sigma_{\mathrm{o}}=\tau_{\mathrm{o}}=\mathrm{o}$, allora dalle (7) si ricaverebbe che è nulla la $M(V, W ; s, t, p+\mathrm{r})$ contro l'ipotesi.

Per dimostrare che le incognite omogenee $\sigma_{0}, \sigma_{1}, \ldots, \sigma_{s-p}, \tau_{0}, \tau_{1}, \ldots, \tau_{t-1}$ 
soddisfano pure al sistema delle $s+t+2-p$ equazioni

$$
\sum_{u=0}^{u=i}(-\mathrm{I})^{u} S_{i-u} \tau_{u}=\sum_{u=0}^{u=i}(-I)^{u} T_{i-.} \sigma_{u} \quad(i=0, r, \ldots, s+t+1-p),
$$

basterd provare per $i \supseteq 2$ che tra le $\sigma_{0}, \sigma_{1}, \ldots, \sigma_{t-p}, \tau_{0}, \tau_{1} \ldots, \tau_{t-p}$ sussiste la $(i+\mathrm{I})^{\text {sima }}$ equazione delle $(8)$, facendo uso dell'ipotesi che tra le $\sigma_{0}, \sigma_{x}, \ldots, \sigma_{s-p}$, $\tau_{0}, \tau_{1}, \ldots, \tau_{t-p}$ sussistano le prime $i$ equazioni delle (8), perchè il sistema delle prime due equazioni delle (8) è identico al sistema delle prime due equazioni delle (7).

$\mathrm{Da}$ questa ipotesi e tenendo conto delle (7) risulta l'equazione

$$
\begin{aligned}
& \sum_{\nu=0}^{v-i=z}(-I)^{v}\left[\sum_{u=0}^{u=v+1} V_{v} \cdot \sigma_{v-u+1}\right] \cdot\left[\sum_{u=0}^{u-i-v-1}(-I)^{u} S_{i-v-u-1} \cdot \tau_{u}\right] \\
= & \sum_{v=0}^{v=i-1}(-I)^{v}\left[\sum_{u=0}^{u=v+1} W_{u} \cdot \tau_{v-u+1}\right] \cdot\left[\sum_{u=0}^{u=i-v-1}(-I)^{u} T_{i-v-u-1} \cdot \sigma_{u}\right],
\end{aligned}
$$

la quale, per mezzo della (2) [ossia semplificando il primo membro per mezzo delle formole

$$
S_{\mathrm{u}}=\sum_{v=0}^{\nu-u-r}(-I)^{u-v-1} S_{v} \cdot V_{u \rightarrow v} \quad(u=1,2,3, \ldots)
$$

ed il secondo membro per mezzo delle formole

diventa :

$$
\left.T_{u}=\sum_{v=0}^{v=u-1}(-1)^{u-v-1} T_{v} \cdot W_{u \sim v} \quad(u=1,2,3, \ldots)\right],
$$$$
\sum_{u=0}^{u-i=1}(-1)^{u}\left[S_{i-u} \sigma_{0}+(-1)^{i-u-1} \sigma_{i-u}\right] \tau_{u}=\sum_{u=0}^{u-i=1}(-1)^{u}\left[T_{i-u} \cdot \tau_{0}+(-1)^{i-u-1} \tau_{i-u}\right] \sigma_{u} .
$$

Siccome semplificando quest'ultima equazione si ottiene la $(i+1)^{\text {sima }}$ equazione delle (8) a meno del fattore di proporzionalita non nullo $\sigma_{0}=\tau_{0}$, si conclude che sussiste il sistema di tutte le $s+t+2-p$ equazioni (8), e quindi è nulla la matrice $M(S, T ; s, t, p)$ e non è nulla la $M(S, T ; s, t, p+1)$, perchè non è $\sigma_{0}=\tau_{0}=0$.

\section{$\int 2$.}

La matrice di Sylvester delle funzioni aleph e la condizione affinchè due equazioni ad una incognita abbiano più radici in comune.

Posto

$$
F=\sum_{i=0}^{i-s+3} a_{i} x^{s+1-i}, \quad F^{\prime}=\sum_{i=0}^{i=t+1} b_{i} x^{i+1-i}
$$

si considerino le due equazioni ad una incognita $x$ :

$$
\begin{aligned}
& F=\text { o, } \\
& F^{\prime}=\text { o, }
\end{aligned}
$$

dei rispettivi gradi $s+\mathrm{I}, t+\mathrm{I}$; senza togliere nulla alla generalita è lecito supporre $a_{0}=b_{0}=\mathrm{I}$. Si designino con $x_{0}, x_{1}, \ldots, x_{s}$ le $s+$ I radici della (ro) e con $x_{0}^{\prime}$, 
$x_{1}^{\prime}, \ldots, x_{t}^{\prime}$ le $t+1$ radici della (Io'); inoltre si faccia la convenzione di porre

$a_{i}=0$, se $i$ è negativo, oppure maggiore di $s+\mathrm{r}$,

$b_{i}=0$, se $i$ è negativo, oppure maggiore di $t+\mathrm{I}$.

Tenendo conto delle notazioni introdotte nel $\$ I, dalla citata formola di P. Gordan (cfr. il teorema enunciato alla fine del $\ 5$ di $\mathfrak{R}$ ) segue:

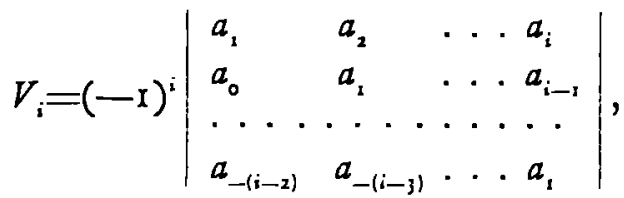

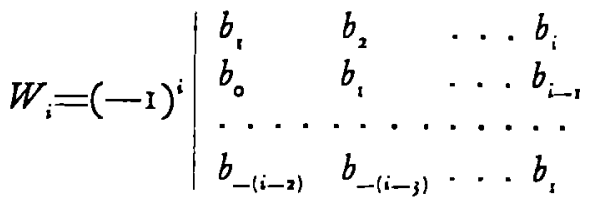

$(i=1,2,3, \ldots)$.

Essendo sempre $0 \leqslant p \leqslant \min (s, t)$, si designi $\operatorname{con} M(a, b ; s, t, p)$ la matrice, che si puó chiamare matrice di Sylvester dei coefficienti, di $s+t+2-2 p$ linee e di $s+t+2-p$ colonne, nella quale gli elementi della colonna $(i+1)^{\text {sima }}$ $(i=0,1, \ldots, s+t+1-p)$ sono

$$
a_{i}, \quad a_{i-1}, \ldots, a_{i-1+p}, \quad b_{i}, \quad b_{i-1}, \ldots, b_{i-s+p} .
$$

Evidentemente si ha:

TeOrema II. - Se è nulla la matrice $M(a, b ; s, t, p)$, è pure nulla la matrice $M(S, T ; s, t, p)$, ed inversamente.

Cosi, la nota proposizione:

Affinche $F=0, F^{\prime}=\mathrm{o}$ abbiano in comune solo $p+\mathrm{I}$ radici, occorre e basta che sia nulla la $M(a, b ; s, t, p)$ ed inoltre, quando è $p<\min (s, t)$, non nullia la $M(a, b ; s, t, p+\mathrm{I})$,

per mezzo dei teoremi I, II si potrà trasformare nel seguente modo:

Teorema III. - Affincbe le equazioni $F=0, F^{\prime}=0$ abbiano in comune solo $p+\mathrm{I}$ radici, occorre e basta che sia nulla la matrice $M(V, W ; s, t, p)$ ed inoltre, quando e $p<\min (s, t)$, non nulla la matrice $M(V, W ; s, t, p+1)$.

Quindi risulta come nella ricerca delle condizioni, affinchè due equazioni ad una incognita abbiano più radici in comune, si possa prendere in considerazione, invece del determinante di Bezout o della matrice di Sylvester dei coefficienti, una nuova matrice simile a questa, gli elementi della quale non sono $i$ coefficienti delle due date equazioni, ma le funzioni aleph delle loro radici, funzioni che si ottengono subito dai coefficienti per mezzo delle (II). Questa nuova matrice $M(V, W ; s, t, p)$ si puó chiamare matrice di Sylvester delle funzioni aleph.

La matrice di Syzvester delle funzioni aleph è importante poi, perchè è suscetribile d'una notevole generalizzazione, che non ha luogo per quella dei coefficienti. Si definirł matrice ampliata di Sylvester delle funzioni aleph e si designerà col simbolo $M(V, W ; s, t, p, n)$ la matrice di $s+t+2-2 p$ linee e di $n+1$ colonne, nella quale gli elementi della colonna $(i+1)^{\operatorname{sima}}(i=0,1, \ldots, n)$ sono

$$
V_{i}, \quad V_{i-1}, \ldots, V_{i-s+p}, \quad W_{i}, \quad W_{i-1}, \ldots, W_{i-i+p} .
$$

Supposto che le equazioni ( 10$)$, ( $\mathrm{Io}^{\prime}$ ) abbiano in comune solo $p+\mathrm{I}$ radici, al- 
lora solo $p+\mathrm{I}$ delle $x_{0}, x_{1}, \ldots, x_{s}$ saranno uguali a $p+\mathrm{I}$ delle $x_{0}^{\prime}, x_{1}^{\prime}, \ldots, x_{i}^{\prime}$; per fissare le idee si abbia

$$
x_{0}=x_{0}^{\prime}, \quad x_{s}=x_{i}^{\prime}, \ldots, x_{p}=x_{p}^{\prime} .
$$

Detta $(T-X)_{i}$ la $T_{i}$, quando si faccia $x_{0}^{\prime}=x_{i}^{\prime}=\cdots=x_{p}^{\prime}=0$, ed osservando che evidentemente

$$
\begin{aligned}
& (T-X)_{0}=\mathrm{r}, \\
& (T-X)_{i}=0, \text { se } i \text { è negativo, oppure maggiore di } t-p,
\end{aligned}
$$

dalla (3) segue :

$$
\left(S_{i}-T_{i}\right)+\sum_{u=1}^{u=t-p} S_{i-u} \cdot(T-X)_{u}-\sum_{u=1}^{u=s-p} T_{i-u} \cdot(S-X)_{u}=0 \quad(i=0,1,2, \ldots, n)
$$

e similmente dalla (6):

$$
\left(V_{i}-W_{i}\right)+\sum_{u=1}^{u=s-p}(-\mathrm{r})^{u} V_{i-u} \cdot(S-X)_{u}-\sum_{u=1}^{u=t-p}(-\mathrm{I})^{u} W_{i-u} \cdot(T-X)_{u}=0 \quad(i=0,1,2, \ldots, n) .
$$

Mentre le (I2) diventano evanescenti, se è $n \geqslant s+t+2-p$, le (I3) invece sono sempre vere equazioni comunque grande sia $n$; onde, eliminando dalle (I3) le $(-\mathrm{I})^{u} \cdot(S-X)_{u}(u=\mathrm{I}, 2, \ldots, s-p)$ e le $(-\mathrm{I})^{u} \cdot(T-X)_{u}(u=\mathrm{I}, 2, \ldots, t-p)$, segue che è nulla la matrice $M(V, W ; s, t, p, n)$, qualunque sia $n$. Siccome poi tale matrice $M(V, W ; s, t, p, n)$ è pure nulla, quando le equazioni (Io), (Io') hanno in comune più di $p+I$ radici, si conclude:

Teorema IV. - Se le equazioni $F=0, F^{\prime}=0$ banno in comune $p+$ I radici, allora è nulla qualsiasi matrice ampliata di SYLVESTER delle funzioni aleph, ossia è nulla la matrice

$$
M(V, W ; s, t, p, N),
$$

dove $N$ ' un intero finito, ma grande quanto si vuole.

$\mathrm{Si}$ considerino ora le funzioni simmetriche caratteristiche (cfr. il $\int$ I di $\mathfrak{N}$ ) delle radici delle due equazioni $F=0, F^{\prime}=0$. Perciò si designi con $\left\{h_{0}, h_{1}, \ldots, h_{s}\right\}_{s}$, essendo $b_{o}, h_{1}, \ldots, b_{\text {s }}$ interi tali che $0 \leqslant b_{0}<b_{s}<\cdots<b_{s}$, la funzione simmetrica caratteristica

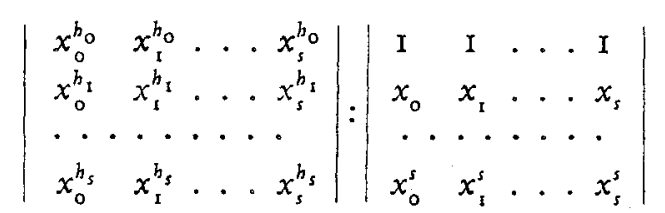

nelle $x_{0}, x_{1}, \ldots, x_{s}$. Quando invece delle $x_{0}, x_{1}, \ldots, x_{s}$ si considerano le $x_{0}^{\prime}, x_{1}^{\prime}, \ldots, x_{t}^{\prime}$, allora si designerà con $\left\{b_{0}^{\prime}, h_{1}^{\prime}, \ldots, b_{l}^{\prime}\right\}_{t}^{\prime}$ la funzione simmetrica caratteristica $\left\{b_{0}^{\prime}, b_{1}^{\prime}, \ldots, b_{t}^{\prime}\right\}_{t}$ nelle $x_{0}^{\prime}, x_{1}^{\prime}, \ldots, x_{i}^{\prime}$.

Applicando la formola del TRUdi (cfr. il $\$ 5$ di $\mathfrak{N}$ ) il teorema IV diventa :

Teorema V. - Essendo $k_{0}, k_{1}, \ldots, k_{s+t+1-2 p}$ interi qualunque soddisfacenti alle disuguaglianze $0 \leq k_{\mathrm{o}}<k_{\mathrm{s}}<\cdots<k_{s+t+1-2 p}$, si chiami $\Pi_{k_{0} k_{1} \ldots k_{s+t+1-2 p}}$ la funzione $\sum \pm\left\{0, \mathrm{I}, \ldots, p-\mathrm{I}, b_{p}, h_{p+s}, \ldots, h_{s}\right\}_{s} \cdot\left\{0, \mathrm{I}, \ldots, p-\mathrm{I}, h_{p}^{\prime}, h_{p+1}^{\prime}, \ldots, b_{t}^{\prime}\right\}_{t}^{\prime}$, dove la sommatoria è estesa a tutti $i$ valori delle $b_{p}, b_{p+1}, \ldots, b_{s}, h_{p}^{\prime}, b_{p+1}^{\prime}, \ldots, b_{i}^{\prime}$, 
per cui $h_{p}, h_{p+1}, \ldots, h_{s}, h_{p}^{\prime}, h_{p+1}^{\prime}, \ldots, b_{1}^{\prime} \dot{e}$ tona qualunque permutazione degli interi $k_{\mathrm{o}}+p, k_{\mathrm{s}}+p, \ldots, k_{\mathrm{s}+t+1-2 \mathrm{p}}+p$, tale che

$$
b_{p}<b_{p+1}<\cdots<b_{s}, \quad b_{p}^{\prime}<b_{p+1}^{\prime}<\cdots<b_{i}^{\prime},
$$

e dove si attribuisce a ciascun termine il segno + , oppure -, secondocbe $h_{p}, b_{p+1}, \ldots, b_{s}$, $b_{p}^{\prime}, b_{p+1}^{\prime}, \ldots, b_{1}^{\prime}$ è una permutazione pari, oppure dispari della serie di numeri $k_{\circ}+p$, $k_{t}+p, \ldots, k_{s \rightarrow l+1-2 p}+p$.

Se le equazioni $F=0, F^{\prime}=0$ banno in comune $p+\mathrm{I}$ radici, allora sono nulle tutte le funzioni

$$
\Pi_{k_{0} k_{1} \ldots k_{s+1+1-2 p}}
$$

\section{$\$ 3$.}

\section{Costruzione del M.C.D. e del M.C.M. di due funzioni algebriche intere di una sola variabile.}

Nell'ipotesi che le equazioni $F=0, F^{\prime}=0$ abbiano in comune solo $p+1$ radici, si considerino delle ( $\mathrm{I}_{2}$ ) e delle ( $\mathrm{I}_{3}$ ) quelle per cui $i=\mathrm{I}, 2, \ldots, s+t+\mathrm{I}-p$, ossia i sistemi :

$$
\begin{gathered}
\left(S_{i}-T_{i}\right)+\sum_{u=1}^{n=t-p} S_{i-u} \cdot(T-X)_{n}-\sum_{u=1}^{u=s-p} T_{i-u} \cdot(S-X)_{u}=0 \quad(i=1,2, \ldots, s+1+1-p) \\
\left(V_{i}-W_{i}\right)+\sum_{u=-1}^{u=s-p}(-I)^{u} V_{i-u} \cdot(S-X)_{u}-\sum_{u=1}^{u-t-p}(-1)^{u} W_{i} \cdot(T-X)_{u}=0 \\
(i=1,2, \ldots, s+t+1-p) .
\end{gathered}
$$

Si chiamino:

$\Delta_{a}(k)[k=0,1, \ldots, \min (s, t)]$ il determinante di $(s+t-2 k+2)^{\text {simo }}$ ordine, nel quale gli elementi della linea $(j+1)^{\operatorname{sim} a}(j=0,1, \ldots, s+t-2 k+1)$ sono:

$$
a_{j}, \quad a_{j-1}, \ldots, a_{j-t+k}, \quad b_{j}, \quad b_{j-1}, \ldots, b_{j-s+k} ;
$$

$\Delta_{V}(k)[k=0, \mathrm{I}, \ldots, \min (s, t)]$ il determinante di $(s+t-2 k+2)^{\text {simo }}$ ordine, nel quale gli elementi della linea $(j+\mathrm{I})^{\mathrm{sima}}(j=0, \mathrm{I}, \ldots, s+t-\mathbf{2} k+\mathrm{I})$ sono:

$$
V_{j}, \quad V_{j-1}, \ldots, V_{j-s+k}, \quad W_{j}, \quad W_{j-1}, \ldots, W_{j-1+k} \text {. }
$$

Dalla citata formola del Trudi (cfr. il $\int \rho$ di $\mathfrak{R}$ ) e da quella di P. Gordan (cfr. il teorema enunciato alla fine del $\oint 5 \mathrm{di} \mathfrak{R}$ ) si ottiene la notevole relazione:

$$
\Delta_{a}(k)=(-1)^{i s-k+1)(t-k+1)} \Delta_{V}(k) \quad\left(k=0,1, \ldots, \min (s, t)^{4}\right) \text {. }
$$

Indicando poi per brevità :

con $M(a, b)_{p}$ la matrice di $s+t-2 p$ linee e di $s+t+1-2 p$ colonne, nella quale gli elementi della linea $j^{\text {sina }}(j=1,2, \ldots, s+t-2 p)$ sono

$$
a_{j}-b_{j}, \quad a_{j-1}, \quad a_{j-2}, \ldots, a_{j-1+p}, \quad b_{j-1}, \quad b_{j-2}, \ldots, b_{j-i+p} \text {; }
$$

4) Di questa relazione si è tenuto conto implicitamente nel $\S 7$ della mia Memoria: Ordine di una varietá piì ampia di quella rappresentata coll'annullare tutti $i$ minori di dato ordine estratti da una data matrice generica di forme [Memorie del R. Istituto Lombardo di Scienze e Lettere, serie $3^{2}$, vol. X (1904), pp. 10I-I34].

Resd. Circ. Matem. Palermo, t, XXV ( $\mathrm{s}^{\circ} \mathrm{sem}$, 1908). - Stampato il 28 novembre 1907. 
con $M(V, W)_{p}$ la matrice di $s+t-2 p$ linee e di $s+t+\mathrm{r}-2 p$ colonne, nella quale gli elementi della linea $j^{\text {sima }}(j=1,2, \ldots, s+t-2 p)$ sono

$$
V_{i}-W_{j}, \quad V_{j-1}, \quad V_{j-2}, \ldots, V_{j-s+p}, \quad W_{j-1}, \quad W_{j-2}, \ldots, W_{j-i+p},
$$

si chiamino:

$D_{a}(k)$ il determinante di $(s+t-2 p)^{\text {simo }}$ ordine ricavato dalla $M(a, b)_{p}$ togliendo in questa la colonna $(k+\mathrm{I})^{\text {sima }}$;

$D_{V}(k)$ il determinante di $(s+t-2 p)^{\text {simo }}$ ordine ricavato dalla $M(V, W)_{p}$ togliendo in questa la colonna $(k+\mathrm{r})^{\operatorname{sima}}$, dove $k=0, \mathrm{r}, \ldots, s+t-2 p$.

Evidentemente i simboli $D_{a}(0), \Delta_{a}(p+\mathrm{I})$ hanno lo stesso significato e cosi pute $D_{V}(\mathrm{o}), \Delta_{V}(p+\mathrm{I})$, onde per la ( $\left.\mathrm{I}_{4}\right)$ si ottiene:

(I5) $D_{a}(\mathrm{o})=\Delta_{a}(p+\mathrm{I})=(-\mathrm{I})^{(s-p)(t-p)} D_{V}(\mathrm{o})=(-\mathrm{I})^{(s-p)(t-p)} \Delta_{V}(p+\mathrm{I})$.

Per l'ipotesi fatta che le $F=o, F^{\prime}=0$ abbiano in comune solo $p+$ I radici, tenendo conto della $6^{a}$ proposizione della importante Nota: Ueber den gemeinsamen Factor zweier binären Formen ${ }^{5}$ ) del Prof. M. Noether, risulta:

$$
\Delta_{a}(0)=\Delta_{a}(\mathrm{I})=\cdots=\Delta_{a}(p)=0, \quad \Delta_{a}(p+\mathrm{I}) \neq 0 .
$$

Quindi, osservando che è nulla la $M(a, b ; s, t, p)$, dalle (I $\left.2^{\prime}\right)$ si ottengono le relazioni :

$$
\begin{array}{rlr}
D_{a}(k) & =\lambda \cdot(T-X)_{k} \quad(k=0, \mathrm{t}, \ldots, t-p), \\
(-\mathrm{I})^{t-p-\mathrm{r}} D_{a}(k) & =\lambda .(S-X)_{k-t+p} \quad(k=t-p+\mathrm{t}, t-p+2, . ., s+t-2 p) ;
\end{array}
$$

e analogamente, osservando che è nulla la $M(V, W ; s, t, p)$, dalle (1 $\left.3^{\prime}\right)$ :

$$
D_{V}(k)=(-1)^{(s-p)(t-p)} \lambda \cdot(S-X)_{k} \quad(k=0, \mathrm{I}, \ldots, s-p),
$$

$$
(-\mathrm{r})^{s-p-1} D_{V}(k)=(-\mathrm{I})^{(s-p)(t-p)} \lambda .(T-X)_{k-s+p}(k=s-p+\mathrm{r}, s-p+2, \ldots, s+t-2 p),
$$

dove il fattore di proporzionalità $\lambda$ non è nullo, essendo per la (I 5$)$ uguale a $\Delta_{a}(p+I)$.

Concludéndo per le (I7), (I6) si puó enunciare:

Teorema VI. - Se le funzioni algebriche intere $F, F^{\prime}$ ammettono come M.C.D. una funzione $\mathbf{\Phi}$ di grado $p+\mathrm{I}$ in $x$, allora le funzioni aleph $Y_{i}(i=\mathrm{I}, 2,3, \ldots)$ di ordine $i$ delle radici dell'equazione in $x \omega=0$ sono date dalle formole:

$$
Y_{i}=\sum_{k=0}^{k=\min (i, s-p)}(-\mathrm{I})^{k} V_{i-k} \frac{D_{V}(k)}{D_{V}(\mathrm{o})}
$$

TeOrema VII. - Se le funzioni algebriche intere $F, F^{\prime}$ ammettono come M.C.M. una funzione $\Psi$ di grado $s+t-p+\mathrm{I}$ in $x$, allora

$$
\Psi=\sum_{i=0}^{i=s+t+\mathrm{I}-p} \sum_{k=0}^{k=\min (i, t-p)}(-\mathrm{I})^{k} a_{i-k} \frac{D_{a}(k)}{D_{a}(\mathrm{o})} x^{s+t+\mathrm{I}-p-i}
$$

Le funzioni aleph $Y_{i}$ si possono evidentemente scrivere sotto forma di quoziente di due determinanti, dei quali il denominatore è $D_{V}(0)$, e cosi pure i coefficienti di $\Psi$

5) Sitzungsberichte der Physikalisch-medicinischen Societăt in Erlangen, Heft XXVII (1895), pp. IIO-IIS. 
si possono scrivere sotto forma di quoziente di due determinanti, dei quali il denominatore è $D_{a}(\mathrm{o})$.

Similmente dalle ( $\left.16^{\prime}\right)$, ( $\left.17^{\prime}\right)$ si ottengono teoremi analoghi a quelli ora enunciati; inoltre, tanto dalle (I6), ( $16^{\prime}$ ) quanto dalle (I7), ( $17^{\prime}$ ) si ottengono subito le funzioni quozienti $F: \Phi, F^{\prime}: \Phi$.

\section{$\$ 4$.}

Risoluzione del problema generale del M.C.D. e del M.C.M. di più funzioni algebriche intere di una sola variabile.

Posto

(18) $F_{1}=\sum_{i=0}^{i=m_{1}+1} a(1 ; i) x^{m_{1}-i+1}, \quad F_{2}=\sum_{i=0}^{i=m_{2}+1} a(2 ; i) x^{m_{2}-i+1}, \ldots, F_{r}=\sum_{i=0}^{i=m_{r}+1} a(r ; i) x^{m_{r}-i+1}$, si considerino le $r$ funzioni algebriche intere di una sola variabile $x$ :

$$
F_{1}, \quad F_{2}, \ldots, F_{r} \text {. }
$$

Senza togliere nulla alla generalita è lecito supporre

$$
a(\mathrm{I} ; 0)=a(2 ; 0)=\cdots=a(r ; 0)=\mathrm{I} ;
$$

inoltre è utile fare la convenzione di attribuire il valore zero alle $a(b ; i)$, per cui $i$ è negativo, oppure maggiore di $m_{h}+\mathrm{I}$, essendo $h$ uno qualunque degli interi $\mathrm{r}, 2, \ldots, r$.

Essendo sempre $b=1,2, \ldots, r$, si designi con $V(b ; i)(i=1,2,3, \ldots)$ la funzione aleph di ordine $i$ delle radici dell'equazione in $x F_{h}=0$, e si convenga di porre

$$
\begin{aligned}
& V(b ; 0)=\mathrm{I}, \\
& V(b ; i)=\mathrm{o}, \text { se } i \text { è negativo. }
\end{aligned}
$$

Per la citata formola di P. Gordan (cfr. il teorema enunciato alla fine del $§ s$ di $\mathfrak{R})$ segue :

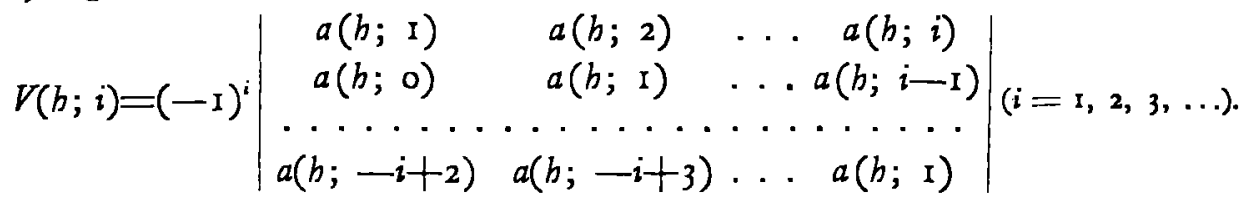

Applicando rispettivamente in modo opportuno i teoremi VI, VII, si risolve subito il problema generale del M.C.D. e del M.C.M. delle funzioni intere $F_{r}, F_{2}, \ldots, F_{r}$, cioè si possono ottenere in funzione delle $a(b ; i)$, o delle $V(b ; i)$, le condizioni ne-

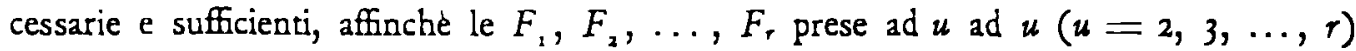
ammettano M.C.D. e M.C.M. di dati gradi.

Essendo $b_{1}, b_{2}, \ldots, b_{u}$ una combinazione qualunque di $u$ degli interi $\mathrm{I}, 2, \ldots, r$ si chiamino:

I $i\left(b_{1}, b_{2}, \ldots, b_{4}\right)+\mathrm{I}$ il dato grado del M.C.D. delle $F_{h_{2}}, F_{h_{2}}, \ldots, F_{h_{4}}$, $2^{\circ} t\left(b_{z}, h_{2}, \ldots, b_{w}\right)+\mathrm{I}$ il dato grado del M.C.M. delle $F_{b_{\mathrm{r}}}, F_{h_{2}}, \ldots, F_{h_{u}}$. Questi numeri $i\left(b_{1}, b_{2}, \ldots, b_{u}\right)$ possono essere interi positivi, oppure uguali a 
zero; o a $-\mathrm{I}$; essi ed i numeri $t\left(h_{1} ; h_{2}, \ldots, h_{u}\right)$ soddisfano ad ovvie disuguaglianze, che per brevità si tralascieranno di scrivere.

E importante invece ricordare le formole fondamentali :

(I9) $\left\{\begin{array}{c}t\left(b_{1}, b_{2}, \ldots, b_{u}\right)=\sum_{b^{\prime}}^{(1)} m_{b_{1}^{\prime}}-\sum_{h^{\prime}}^{(2)} i\left(b_{1}^{\prime}, b_{2}^{\prime}\right)+\ldots \\ \ldots+(-1)^{u-2} \sum_{b^{\prime}}^{(u-1)} i\left(b_{1}^{\prime}, b_{2}^{\prime}, \ldots, b_{u-1}^{\prime}\right)+(-1)^{u-1} i\left(b_{1}, b_{2}, \ldots, b_{u}\right),\end{array}\right.$
(20) $\left\{\begin{array}{c}i\left(b_{1}, b_{2}, \ldots, b_{u}\right)=\sum_{h^{\prime}}^{(1)} m_{h_{1}^{\prime}}^{\prime}-\sum_{b^{\prime}}^{(2)} t\left(b_{1}^{\prime}, b_{2}^{\prime}\right)+\ldots \\ \ldots+(-1)^{u-2} \sum_{h^{\prime}}^{(u-1)} t\left(b_{1}^{\prime}, b_{2}^{\prime}, \ldots, b_{u-1}^{\prime}\right)+(-1)^{u-1} t\left(b_{1}, b_{2}, \ldots, b_{u}\right),\end{array}\right.$ dove $\sum_{h^{\prime}}^{(v)}(v=\mathrm{I}, 2, \ldots, u-\mathrm{I})$ significa che la sommatoria è estesa a tutte le combinazioni $h_{1}^{\prime}, b_{2}^{\prime}, \ldots, h_{v}^{\prime}$ di $v$ delle $h_{1}, h_{2}, \ldots, h_{u}$.

Quindi per le (19), (20) segue che tra i vari modi di risolvere il problema ge-

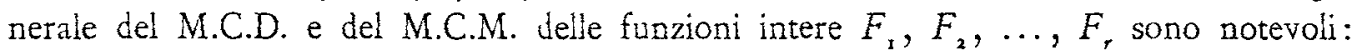

il metodo del M.C.D., cioé l'applicazione ripetuta del solo teorema VI,

il metodo del M.C.M., cióe l'applicazione ripetuta del solo teorema VII.

\section{Metodo del M.C.D.}

Nel definire $i$ seguenti simboli occorre l'ipotesi che nessuna delle $i\left(b_{1}, b_{2}, \ldots, b_{u}\right)$ sia negativa.

Essendo $b_{1}, b_{2}$ una combinazione qualunque di due degli interi $\mathrm{I}, 2, \ldots, r$, si chiamino:

$I^{\circ} \Delta_{V}\left(b_{1}, h_{2} ; k\right)\left[k=0, \mathrm{r}, \ldots, i\left(h_{1}, h_{2}\right)+\mathrm{I}\right]$ il determinante di $\left(m_{h_{1}}+m_{h_{2}}-2 k+2\right)^{\text {simo }}$ ordine, nel quale gli elementi della linea $(j+\mathrm{I})^{\text {simia }}\left(j=0, \mathrm{I}, \ldots, m_{b_{1}}+m_{h_{2}}-2 k+\mathrm{I}\right)$ sono:

$V\left(h_{1} ; j\right), V\left(b_{1} ; j-I\right), \ldots, V\left(h_{1} ; j-m_{h_{1}}+k\right), V\left(h_{2} ; j\right), \quad V\left(h_{2} ; j-1\right), \ldots, V\left(h_{2} ; j-m_{h_{2}}+k\right)$;

$2^{\circ} D_{V}\left(h_{1}, h_{2} ; k\right)\left[k=\mathrm{I}, 2, \ldots, m_{h_{5}}-i\left(h_{\mathrm{r}}, b_{2}\right)\right]$ il determinante di $\left[m_{h_{1}}+m_{b_{2}}-2 i\left(b_{\mathrm{r}}, h_{2}\right)\right]^{\text {simo }}$ ordine, nel quale gli elementi della linea $(j+1)^{\operatorname{sima}}\left[j=1,2, \ldots, m_{h_{1}}+m_{h_{2}}-2 i\left(b_{1}, h_{2}\right)\right]$ sono:

$$
V\left(b_{1}, j\right)-V\left(b_{2}, j\right), \quad V\left(b_{1} ; j-1\right), \ldots, V\left(b_{1} ; j-k+1\right)
$$

$V\left(h_{1} ; j-k-\mathrm{I}\right), \ldots, V\left(b_{1} ; j-m_{h_{1}}+i\left(h_{1}, h_{2}\right)\right), \quad V\left(b_{2} ; j-\mathrm{I}\right), \ldots, V\left(b_{2} ; j-m_{b_{2}}+i\left(b_{1}, b_{2}\right)\right)$; e si convenga di porre

$$
D_{V}\left(b_{1}, h_{2} ; 0\right)=\Delta_{V}\left(b_{1}, b_{2} ; i\left(h_{1}, b_{2}\right)+1\right) .
$$

Più generalmente, essendo $b_{1}, b_{2}, \ldots, b_{u}$ una combinazione qualunque di $u$ degli interi $\mathrm{I}, 2, \ldots, r$, si chiamino:

$$
\text { I }^{\text {o }} \Delta_{V}\left(b_{1}, \ldots, b_{u} ; k\right)\left[k=0, I, \ldots, i\left(h_{1}, \ldots, b_{u}\right)+\mathrm{I}\right] \text { il determinante di }
$$
$\left[i\left(h_{1}, \ldots, b_{u-1}\right)+m_{h_{u}}-2 k+2\right]^{\text {simo }}$ ordine, nel quale gli elementi della linea $(j+\mathrm{I})^{\text {sima }}\left[j=0, \mathrm{x}, \ldots, i\left(h_{\mathrm{r}}, \ldots, h_{u-1}\right)+m_{h_{u}}-2 k+\mathrm{I}\right)$ sono:

$$
\begin{gathered}
V\left(b_{1}, \ldots, h_{u-1} ; j\right), \quad V\left(b_{1}, \ldots, b_{u-1} ; j-1\right), \ldots, V\left(b_{1}, \ldots, b_{u-1} ; j-i\left(b_{1}, \ldots, b_{u-1}\right)+k\right), \\
V\left(h_{u} ; j\right), \quad V\left(h_{u} ; j-1\right), \ldots, V\left(b_{u} ; j-m_{h_{4}}+k\right) ;
\end{gathered}
$$

$2^{\circ} D_{V}\left(b_{1}, \ldots, b_{u} ; k\right)\left[k=\mathrm{I}, 2, \ldots, i\left(b_{1}, \ldots, b_{u-1}\right)-i\left(h_{1}, \ldots, h_{u}\right)\right]$ il determinante di $\left[i\left(b_{1}, \ldots, b_{u-1}\right)+m_{h_{\mu}}-2 i\left(b_{1}, \ldots, b_{u}\right)\right]^{\text {simo }}$ ordine, nel quale gli 
elementi della linea $(j+\mathrm{I})^{\text {sima }}\left[j=\mathrm{I}, 2, \ldots, i\left(b_{1}, \ldots, b_{u-s}\right)+m_{h_{u}}-2 i\left(b_{1}, \ldots, h_{u}\right)\right]$ sono:

$V\left(h_{1}, \ldots, b_{u-1} ; j\right)-V\left(b_{u} ; j\right), \quad V\left(b_{1}, \ldots, b_{u-1} ; j-1\right), \ldots, V\left(b_{1}, . ., b_{u-1} ; j-k+1\right)$ $V\left(h_{1}, \ldots, b_{u-1} ; j-k-1\right), \ldots, V\left(b_{1}, \ldots, b_{u-1} ; j-i\left(b_{1}, \ldots, h_{u-1}\right)+i\left(h_{1}, \ldots, b_{u}\right)\right)$, $V\left(b_{u} ; j-1\right), \ldots, V\left(h_{u} ; j-m_{h_{u}}+i\left(b_{1}, \ldots, b_{u}\right)\right)$.

Si convenga poi di porre

$$
\begin{aligned}
& \text { I } \quad D_{V}\left(b_{s}, \ldots, b_{u} ; 0\right)=\Delta_{V}\left(b_{1}, \ldots, b_{u} ; i\left(b_{1}, \ldots, b_{u}\right)+1\right) \text {, } \\
& 2^{0} V\left(h_{1}, \ldots, h_{u} ; j\right)=\sum_{k=0}^{k=\pi \min \left[j, i\left(h_{1}, \ldots, h_{u-1}\right)-i\left(h_{1}, \ldots, b_{u}\right)\right]}(-\mathrm{I})^{k} V\left(h_{1}, \ldots, k_{u-1} ; j-k\right) \frac{D_{V}\left(h_{1}, \ldots, h_{u} ; k\right)}{D_{V}\left(b_{x}, \ldots, h_{u} ; 0\right)} \\
& (j=0,1,2, \ldots), \\
& V\left(b_{1}, \ldots, b_{u} ; j\right)=0 \text {, se } j \text { è negativo, }
\end{aligned}
$$

attribuendo il valore $m_{h_{1}}$ al simbolo $i\left(b_{1}, \ldots, h_{u_{-}}\right)$, quando $u=2{ }^{6}$ ).

Applicando ripetutamente il teorema VI si conclude:

Teorema VIII. - Affincbè le funzioni algebriche intere $F_{1}, F_{2}, \ldots, F_{r}$ della sola variabile $x$ siano tali che, essendo $h_{1}, b_{2}, \ldots, b_{u}(u=2,3, \ldots, r)$ una combinazione qualunque di $u$ degli interi $\mathrm{I}, 2, \ldots, r$, il M.C.D. di $F_{h_{1}}, F_{h_{2}}, \ldots, F_{h_{u}}$ risulti di grado $i\left(b_{1}, \ldots, h_{n}\right)+1$, occorre e basta che siano nulli tutti $i$ determinanti $e$ non nulli tutti $i$ determinanti

$$
\Delta_{\nu}\left(b_{z}, \ldots, h_{u} ; k\right) \quad\left[k=0,1, \ldots, i\left(b_{1}, \ldots, b_{u}\right)\right]
$$

$$
s_{V}\left(h_{1}, \ldots, h_{n} ; i\left(b_{1}, \ldots, b_{u}\right)+\mathrm{I}\right)
$$

dove nelle (2I), (22) $h_{1}, h_{2}, \ldots, b_{u}$ variano in modo da ottenere tutte quelle combinazioni di $u$ degli interi $\mathrm{I}, 2, \ldots, r$, per cui non sia $i\left(b_{1}, \ldots, b_{\mathrm{a}}\right)=-\mathrm{I}$.

Inoltre, se non $\dot{e} i\left(b_{1}, \ldots, h_{u}\right)=-\mathrm{I}$, allora le corrispondenti $V\left(b_{1}, \ldots, h_{u} ; j\right)(j=1,2,3, \ldots)$ sono le funzioni aleph delle radici dell'equazione ottenuta ugnagliando a zero il M.C.D. delle $F_{h_{1}}, F_{h_{2}}, \ldots, F_{h_{4}}$.

\section{Metodo del M.C.M.}

Nel definire i seguenti simboli occorre l'ipotesi che le $t\left(b_{1}, b_{2}, \ldots, b_{u}\right)$ siano tali che non risulti

$$
t\left(h_{i}, \ldots, b_{u}\right)=t\left(b_{1}, \ldots, h_{u-1}\right)+m_{b_{u}}+\mathrm{I},
$$

dove qui e nel seguito si fa la convenzione di attribuire il valore $m_{h_{\mathbf{z}}}$ al simbolo $t\left(b_{1}, \ldots, b_{u-1}\right)$, quando $u=2$.

Essendo $b_{1}, b_{2}$ una combinazione qualunque di due degli interi $r, 2, \ldots, r$ si chiamino:

$$
I^{\circ} \Delta_{a}\left(h_{1}, h_{2} ; k\right)\left[k=0, \mathrm{I}, \ldots, m_{k_{1}}+m_{h_{2}}-t\left(h_{1}, h_{2}\right)+\mathrm{I}\right] \text { il determinante }
$$

6) Nell'applicare le relazioni che servono a calcolare le $V\left(h_{1}, \ldots, h_{w} ; k\right)$ sard in generale utile, per abbreviare i calcoli, disporre le $h_{1}, h_{2}, \ldots, h_{*}$ in modo, che risulti

$$
i\left(h_{1}, \ldots, h_{u-1}\right) \leqslant i\left(h_{1}^{\prime}, \ldots, h_{u-1}^{\prime}\right),
$$

essendo $h_{1}^{\prime}, b_{2}^{\prime}, \ldots, h_{a-1}^{\prime}$ una qualunque combinazione delle $b_{1}, b_{2}, \ldots, h_{a}$. 
di $\left(m_{h_{1}}+m_{h_{2}}-2 k+2\right)^{\text {simo }}$ ordine, nel quale gli elementi della linea $(j+\mathrm{I})^{\text {sima }}$ ( $\left.j=0, \mathrm{I}, \ldots, m_{h_{1}}+m_{h_{2}}-2 k+\mathrm{I}\right)$ sono:

$a\left(b_{1} ; j\right), \quad a\left(b_{1} ; j-1\right), \ldots, a\left(b_{1} ; j-m_{h_{2}}+k\right), a\left(b_{2} ; j\right), a\left(b_{2} ; j-1\right), \ldots, a\left(b_{2} ; j-m_{h_{1}}+k\right)$;

$2^{\circ} D_{a}\left(h_{1}, h_{2} ; k\right)\left[k=\mathrm{I}, 2, \ldots, t\left(h_{1}, h_{2}\right)-m_{h_{1}}\right]$ il detern. di $\left[2 . t\left(h_{1}, h_{2}\right)-m_{h_{1}}-m_{h_{2}}\right]^{\text {simo }}$ ordine, nel quale gli elementi della linea $(j+\mathrm{I})^{\text {sima }}\left[j=1,2, \ldots, 2 . t\left(b_{1}, h_{2}\right)-m_{h_{1}}-m_{h_{2}}\right]$ sono:

$$
\begin{gathered}
a\left(h_{1} ; j\right)-a\left(h_{2} ; j\right), a\left(h_{1} ; j-1\right), \ldots, a\left(h_{1} ; j-k+1\right), a\left(h_{1} ; j-k-1\right), \ldots, \\
a\left(h_{1} ; j-t\left(h_{1}, h_{2}\right)+m_{h_{1}}\right), a\left(h_{2} ; j-1\right), \ldots, a\left(h_{2} ; j-t\left(h_{1}, h_{2}\right)+m_{h_{2}}\right) ;
\end{gathered}
$$

e si convenga di porre:

$$
D_{a}\left(b_{1}, b_{2} ; 0\right)=\Delta_{a}\left(b_{1}, h_{2} ; m_{b_{1}}+m_{h_{2}}-t\left(b_{1}, b_{2}\right)+1\right) .
$$

Più generalmente, essendo $b_{1}, b_{2}, \ldots, b_{u}$ una combinazione qualunque di $u$ degli interi $r, 2, \ldots, r$, si chiamino:

$\mathrm{I}^{\circ} \Delta_{a}\left(b_{1}, \ldots, h_{u} ; k\right)\left[k=0, \mathrm{I}, \ldots, t\left(h_{1}, \ldots, h_{u-1}\right)+m_{h_{u}}-t\left(h_{1}, \ldots, h_{u}\right)+\mathrm{I}\right]$ il determinante di $\left[t\left(b_{1}, \ldots, h_{u-1}\right)+m_{h_{u}}-2 k+2\right]^{\text {sino }}$ ordine, nel quale gli elementi della linea $(j+\mathrm{I})^{\text {sina }}\left[j=0, \mathrm{I}, \ldots, t\left(b_{1}, \ldots, h_{u-1}\right)+m_{h_{u}}-2 k+\mathrm{I}\right]$ sono: $a\left(b_{1}, \ldots, b_{u-1} ; j\right), \quad a\left(b_{1}, \ldots, h_{u-1} ; j-1\right), \ldots, a\left(b_{1}, \ldots, b_{u-1} ; j-m_{h_{u}}+k\right)$, $a\left(h_{u} ; j\right), \quad a\left(h_{u} ; j-1\right), \ldots, a\left(h_{u} ; j-t\left(h_{1}, \ldots, h_{u-1}\right)+k\right)$;

$2^{\circ} D_{a}\left(b_{1}, \ldots, b_{u} ; k\right)\left[k=\mathrm{I}, 2, \ldots, t\left(h_{1}, \ldots, h_{u}\right)-t\left(b_{1}, \ldots, h_{u-t}\right)\right]$ il determinante $\operatorname{di}\left[2 . t\left(b_{1}, \ldots, b_{j}\right)-t\left(b_{1}, \ldots, b_{u-1}\right)-m_{b_{u}}\right]^{\text {simo }}$ ordine, nel quale gli elementi della linea $(j+\mathrm{I})^{\text {sima }}\left[j=\mathrm{I}, 2, \ldots, 2 . t\left(b_{1}, \ldots, b_{u}\right)-t\left(b_{1}, \ldots, h_{u-1}\right)-m_{h_{u}}\right]$ sono :

$$
\begin{gathered}
a\left(h_{1}, \ldots, b_{u-1} ; j\right)-a\left(b_{u} ; j\right), \quad a\left(b_{1}, \ldots, b_{u-1} ; j-1\right), \ldots, a\left(b_{1}, \ldots, b_{u-1} ; j-k+\mathrm{r}\right), \\
a\left(h_{1}, \ldots, b_{u-1}^{\prime} ; j-k-\mathrm{I}\right), \ldots, a\left(b_{1}, \ldots, b_{u-1} ; j-t\left(b_{1}, \ldots, b_{u}\right)+t\left(b_{1}, \ldots, b_{u-1}\right)\right), \\
a\left(h_{n} ; j-\mathrm{I}\right), \ldots, a\left(b_{u} ; j-t\left(b_{t}, \ldots, b_{u}\right)+m_{h_{u}}\right) .
\end{gathered}
$$

Si convenga poi di porre:

$$
\begin{aligned}
& I^{\circ} D_{a}\left(h_{1}, \ldots, b_{u} ; 0\right)=\Delta_{a}\left(b_{1}, \ldots, b_{u} ; t\left(h_{1}, \ldots, b_{u-1}\right)+m_{h_{u}}-t\left(b_{1}, \ldots, b_{u}\right)+\mathrm{I}\right) \text {, } \\
& 2^{\circ} a\left(h_{1}, \ldots, h_{u} ; j\right)=\sum_{k=0}^{k=\min \left[j, t\left(b_{1}, \ldots, h_{u}\right)\right.}(-1)^{\left.-t\left(h_{3}, \ldots, h_{u}-1\right)\right]} a\left(b_{1}, \ldots, b_{u-1} ; j-k\right) \frac{D_{a}\left(b_{1}, \ldots, h_{u} ; k\right)}{D_{a}\left(b_{1}, \ldots, b_{u} ; 0\right)} \\
& {\left[j=0,1,2, \ldots, t\left(h_{1}, \ldots, h_{u}\right)+\mathrm{r}\right] \text {, }}
\end{aligned}
$$

$a\left(b_{1}, \ldots, b_{u} ; j\right)=0$, se $j$ è negativo, oppure se è $\left.j>t\left(b_{1}, \ldots, h_{u}\right)+{ }^{7}{ }^{7}\right)$.

Applicando ripetutamente il teorema VII si conclude:

TeOrema IX. - Affinche le funzioni algebriche intere $F_{\mathrm{t}}, F_{2}, \ldots, F_{\mathrm{r}}$ della sola variabile $x$ siano tali che, essendo $b_{1}, b_{2}, \ldots, h_{u}(u=2,3, \ldots, r)$ una combinazione qualunque di u degli interi $\mathrm{I}, 2, \ldots, r$, il M.C.M. di $F_{h_{1}}, F_{h_{2}}, \ldots, F_{h_{u}}$ risulti di

7) Rispetto alla disposizione pita conveniente delle $h_{t}, \ldots, b_{u}$ nel calcolo delle $a\left(h_{1}, \ldots, b_{u} ; j\right)$ si puo fare un'osservazione analoga a quella fatta per il calcolo del $V\left(h_{1}, \ldots, h_{4} ; j\right)$ nella nota ${ }^{6}$ ). 
grado $t\left(h_{1}, . ., h_{u}\right)+\mathrm{I}$, occorre e basta che siano nulli tutti $i$ determinanti $\Delta_{a}\left(h_{1}, \ldots, h_{u} ; k\right) \quad\left[k=0, \mathrm{I}, \ldots, t\left(h_{1}, \ldots, h_{u-1}\right)+m_{b_{u}}-t\left(h_{1}, \ldots, h_{u}\right)\right]$ e non nulli tutti $i$ determinanti

$$
\Delta_{a}\left(h_{1}, \ldots, h_{u} ; t\left(h_{1}, \ldots, h_{u-1}\right)+m_{h_{u}}-t\left(b_{1}, \ldots, b_{u}\right)+\mathrm{I}\right) \text {, }
$$

dove nelle $(23),(24) b_{1}, h_{2}, \ldots, b_{n}$ variano in modo da ottenere tutte quelle combinazioni di $u$ degli interi $\mathrm{r}, 2, \ldots, r$, per cui non sia $t\left(h_{1}, \ldots, b_{u}\right)=t\left(b_{1}, \ldots, h_{u-1}\right)+m_{h_{u}}+\mathrm{r}$.

Inoltre, se non è $t\left(b_{1}, \ldots, b_{u}\right)=t\left(b_{1}, \ldots, h_{u-1}\right)+m_{b_{u}}+\mathrm{I}$, allora

$$
\sum_{j=0}^{j=\ell\left(h_{1}, \ldots, h_{u}\right)+1} a\left(h_{1}, \ldots, h_{u} ; j\right) x^{t\left(b_{1}, \ldots, h_{u}\right)-j+1}
$$

è il M.C.M. delle $F_{h_{1}}, F_{h_{2}}, \ldots, F_{h_{u}}{ }^{8}$ ).

\section{Casi particolari notevoli.}

I teoremi generali VIII, IX, diventano più semplici, quando si fanno alcune ipotesi particolari per i numeri $i\left(b_{1}, \ldots, b_{n}\right)$ e $t\left(h_{1}, \ldots, b_{n}\right)$.

Infatti quando si suppone che i numeri $i\left(b_{1}, \ldots, b_{u}\right)$, qualunque sia $u$ e comunque si scelgano le $h_{1}, \ldots, b_{u}$, siano tutti uguali tra loro, allora si puó modificare il teorema VIII cosi :

Teorema X. - Affinchè le funioni algebriche intere $F_{1}, F_{2}, \ldots, F_{r}$ della sola variabile $x$, comunque prese ad $u$ ad $u(u=2,3, \ldots, r)$, ammettano sempre uno stesso M.C.D. di grado $p+\mathrm{I}$, essendo $p \gg 0$, occorre e basta che siano nulli tutti $i$ determinanti

e non nulli tutti $i$ determinanti

$$
\Delta_{V}\left(b_{1}, h_{2} ; k\right) \quad(k=0, \mathrm{I}, \ldots, p)
$$

$$
\Delta_{V}\left(b_{1}, h_{2} ; p+\mathrm{I}\right)
$$

dove nelle $(25),(26) h_{1}, h_{2}$ variano in modo da ottenere tutte le combinazioni di due degli interi $\mathrm{I}, 2, \ldots, r$, ed inoltre che siano soddisfatte le relazioni.

onde segue:

$$
V(\mathrm{I}, 2 ; j)=V(\mathrm{I}, 3 ; j)=\cdots=V(\mathrm{r}, r ; j) \quad(j=\mathrm{I}, 2, \ldots, p+\mathrm{r})
$$

$$
\begin{gathered}
V(\mathrm{1}, 2 ; j)=V(\mathrm{x}, 3 ; j)=\cdots=V(\mathrm{1}, r ; j)=V(2,3 ; j)=\cdots=V(2, r ; j)=\cdots=V(r-\mathrm{I}, r ; j) \\
(j=\mathrm{x}, 2, \ldots, p+\mathrm{1}) .
\end{gathered}
$$

Le $V(\mathrm{I}, 2 ; j)(j=\mathrm{I}, 2,3, \ldots)$ sono le funzioni aleph delle radici dell'equazione ottenuta uguagliando a zero il M.C.D. delle $F_{1}, F_{2}, \ldots, F_{r}$.

Se si suppone invece che $\mathrm{i}$ numeri $t\left(b_{1}, \ldots, b_{u}\right)$, qualunque sia $u$ e comunque si scelgano le $b_{1}, \ldots, b_{u}$, siano tutti uguali tra loro, allora si puó modificare il teorema IX cosi :

Teorema XI. - Affinche le funzioni algebriche intere $F_{1}, F_{2}, \ldots, F_{r}$ della sola variabile $x$, comunque prese ad $u$ ad $u(u=2,3, \ldots, r)$, ammettano sempre uno stesso M.C.M. di grado $m+\mathrm{I}$, essendo $m$ non maggiore della somma di due qualunque delle

8) Per convenzione $t\left(h_{1}, \ldots, h_{u-1}\right)=m_{h_{\mathfrak{l}}}$, se $u=2$ 
$m_{1}, m_{2}, \ldots, m_{r}$, occorre e basta che siano nulli tutti $i$ determinanti

$$
\lrcorner_{a}\left(h_{1}, h_{2} ; k\right) \quad\left(k=0,1, \ldots, m_{b_{1}}+m_{h_{2}}-m\right)
$$

e non nulli tutti $i$ determinanti

$$
\Delta_{a}\left(b_{1}, b_{2} ; m_{b_{1}}+m_{h_{2}}-m+\mathrm{r}\right)
$$

dove nelle $(27),(28) h_{1}, h_{2}$, variano in modo da ottenere tutte le combinazioni di due degli interi $\mathrm{r}, 2, \ldots, r$, ed inoltre che siano soddisfatte le relazioni

onde segue:

$$
a(1,2 ; j)=a(1,3 ; j)=\cdots=a(1, r ; j) \quad(j=1,2, \ldots, m+1),
$$

$$
\begin{gathered}
a(\mathrm{x}, 2 ; j)=a(\mathrm{I}, 3 ; j)=\cdots=a(\mathrm{I}, r ; j)=a(2,3 ; j)=\cdots=a(2, r ; j)=\cdots=a(r-\mathrm{r}, r ; j) \\
\quad(j=\mathrm{I}, 2, \ldots, m+\mathrm{r}) .
\end{gathered}
$$

$$
\sum_{j=0}^{j=0} a(1,2 ; j) x^{m-j+1}
$$

è il M.C.M. delle $F_{1}, F_{2}, \ldots, F_{r}$.

Da ultimo é útile osservare che $\mathrm{i}$ risultati generali dei teoremi VIII e IX si potrebbero forse semplificare qualora si spingesse ulteriormente, in modo opportuno, la teoria delle funzioni simmetriche caratteristiche.

Torino, I agosto 1907 .

Giovani Z. Giambeli. 